\title{
Hydromagnetic Instability in a Stellarator
}

\author{
By M. D. Kruskal,* J. L. Johnson, † M. B. Gottlieb* and L. M. Goldman†
}

Kruskal and Tuck ${ }^{1}$ (in a paper hereafter referred to as KT) have examined the influence of a longitudinal magnetic field on the instabilities of the pinch effect. The pinch effect is the confinement of a thin column of plasma by means of the magnetic field due to a high current discharge along the column. Instabilities in the form of lateral "buckling" of the column (in the absence of a longitudinal field) have been predicted theoretically ${ }^{2}$ and are well known experimentally.

In $\mathrm{KT}$ it was noted that when there is a uniform externally imposed longitudinal field much larger than the field of the discharge current, one should expect instabilities in the form of a lateral displacement of the plasma column into a helix of large pitch. At the wavelength of fastest growth the $e$-folding time approximates the time it takes a sound wave in the plasma to traverse the radius of the plasma column. In successive sections we (a) re-examine this problem under the conditions which might be expected to occur in the stellarator during ohmic heating, including the presence of external conductors; $(b)$ apply this theory to the stellarator; $(c)$ show that the external conductors are in fact unimportant; $(d)$ discuss the important effects due to the finite length of the machine; (e) consider the effects of more general current distributions; and $(f)$ give the relevant experimental results.

It should be emphasized that the considerations of this paper apply only to stellarators in which the rotational transform ${ }^{3}$ results from the large scale geometry of the tube (such as a Figure 8 shape) rather than from small local perturbation coils (such as helical windings). It is perhaps worth noting that the theoretical results of the following four sections are given in less condensed form elsewhere; ${ }^{4}$ the appearance of instability and the dependence of the critical current, both on the confining field and on the direction of the plasma current, were predicted in this earlier work well in advance of the experimental confirmation.

\section{INFINITE CYLINDER THEORY}

We start with the analysis of pinch instability under the conditions considered in $\mathrm{KT}$, but now

\footnotetext{
* Project Matterhorn, Princeton University.

+ Project Matterhorn, on loan from Westinghọuse Electric Corp.

+ Now with General Electric Co.
}

additionally taking into account the effect of a thin cylindrical sheet conductor coaxial with the plasma. Familiarity with $\mathrm{KT}$ is assumed.

The material pressure, density and velocity of the plasma are denoted by $p, \rho$, and $\mathbf{v}$, the magnetic and electric fields by $\mathbf{B}$ and $\mathbf{E}$, the current and charge densities by $\mathbf{j}$ and $\epsilon$, the permeability and permittivity of space by $\mu_{0}$ and $\kappa_{0}$, and the ratio of specific heats by $\gamma$. (We employ MKS units throughout.) The equations we use for the interior of the plasma (treated as infinitely conductive) are Eqs. (1) through (8) of KT.

At an interface between plasma and vacuum, $\mathbf{n}$ denotes the unit normal to the surface, directed into the plasma; $\mu$, the normal velocity of the surface; $j^{*}$ and $\epsilon^{*}$, the surface current density and surface charge density; brackets, the jump in the enclosed quantity upon crossing the surface from the vacuum into the plasma; a bar under a quantity, the arithmetic mean of the values of that quantity on each side of the surface; and subscripts $P$ and $V$, respective values on the plasma and vacuum sides of the interface. The equations we use at the interface are Eqs. (9) through (14) of KT.

Suppose we have a sheet of solid material of small thickness, $\delta$, fixed in space with vacuum on both sides. Let $\sigma$ be the volume conductivity of the material and $\tau$ a characteristic time for the phenomena to be considered. If $\delta$ is much less than the penetration distance $\left(\tau / \mu_{0} \sigma\right)^{\frac{1}{3}}$ of the material, the thickness may be disregarded and the sheet treated as a surface of surface conductivity $\sigma^{*}=\sigma^{\delta}$. With the same notation as at an interface, our equations at the sheet are then

$$
\begin{aligned}
\mathbf{n} \times[\mathbf{B}] & =\mu_{0} \mathbf{j}^{*}, \\
\mathbf{n} \cdot[\mathbf{B}] & =0, \\
\mathbf{n} \times[\mathbf{E}] & =0, \\
\mathbf{n} \cdot[\mathbf{E}] & =\epsilon^{*} / \boldsymbol{\kappa}_{0}, \\
\mathbf{E}-\mathbf{n n} \cdot \mathbf{E} & =\mathbf{j}^{*} / \sigma^{*},
\end{aligned}
$$

We use cylindrical coordinates $r, \theta, z$. Consider the following situation (Fig. 1). Inside the infinite cylinder $r=r_{0}$ we have a uniform plasma with $p=p_{0}, \rho=\rho_{0}$, $\mathbf{v}=0, B_{r}=B_{\theta}=0, B_{z}=B_{\mathbf{P}}, \mathbf{E}=0, \mathbf{j}=0, \epsilon=0$. Outside the cylinder $r=r_{0}$ is a vacuum in which $B_{r}=0, \quad B_{\theta}=B_{0} \gamma_{0} / r, \quad B_{z}=B_{\mathrm{V}}, \quad \mathbf{E}=0$. On the 217 
$j_{z}^{*}=j_{0}^{*}, \epsilon^{*}=0$. At $r=r_{1}>r_{0}$ there is a fixed cylindrical thin material sheet of surface conductivity $\sigma^{*}$ on which $j^{*}=0, \epsilon^{*}=0$. This will be an equilibrium situation (time-independent solution) if the constants $\gamma_{0}, \gamma_{1}, p_{0}, \rho_{0}, B_{0}, B_{\mathbf{P}}, B_{\mathrm{V}}, j_{0}^{*}, j_{1}^{*}$, and $\sigma^{*}$ satisfy

$$
\begin{gathered}
B_{0}=\mu_{0} j_{0}^{*}, \quad B_{\mathrm{P}}-B_{\mathrm{V}}=\mu_{0} j_{1}^{*} \\
B_{0}{ }^{2}+B_{\mathrm{V}^{2}}-B_{\mathrm{P}^{2}}=2 \mu_{0} p_{0} .
\end{gathered}
$$

We now seek solutions of our equations which are close to the equilibrium solution just described. We suppose that every physical quantity is equal to its equilibrium value plus a small perturbation term. We consider all our equations as equations for these perturbation quantities and linearize them in the usual way. We obtain a system of algebraic and differential equations, linear and homogeneous, with $r, \theta, z$, and $t$ as independent variables. The coefficients are obviously independent of $\theta, z$, and $t$. Any solution of the equations may therefore be obtained as a superposition of elementary solutions, an elementary solution being one in which each dependent variable is a function of $r$ only (or, in the case of sheet quantities, a constant) multiplied by $\exp (i m \theta+i k z+\omega t)$, where $m, k$, and $\omega$ are constants, the characteristic constants of the elementary solution. We may therefore restrict ourselves to a search for the elementary solutions. To make physical sense we must require that $m$ be an integer and that $k$ be real. Without loss of generality we may assume that $m$ is non-negative.

We next change our notation, each symbol which originally denoted a physical quantity now denoting the coefficient of the exponential in the representation of the perturbation of that quantity. Our equations become linear homogeneous algebraic and ordinary differential equations for these coefficients. We introduce the constants

$$
\begin{gathered}
c^{2}=1 / \mu_{0} \kappa_{0}, \quad s^{2}=\gamma p_{0} / \rho_{0}, \quad h^{2}=B_{P^{2}} / \mu_{0} \rho_{0} \\
\xi^{2}=k^{2}+\frac{\omega^{2}}{s^{2}}, \quad \eta^{2}=k^{2}+\frac{\omega^{2}}{c^{2}}+\frac{\omega^{2}}{h^{2}}, \\
\zeta^{2}=k^{2}+\frac{\omega^{2}}{s^{2}}+\frac{\omega^{2}}{h^{2}}, \quad \psi^{2}=k^{2}+\frac{\omega^{2}}{c^{2}}
\end{gathered}
$$

where $c, s$, and $h$ are the velocities of light, sound, and hydromagnetic waves, respectively. The general

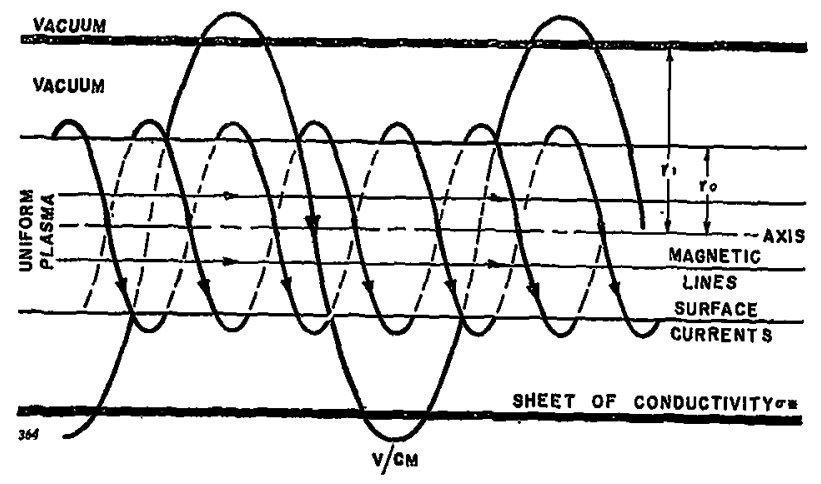

Figure 1. Equilibrium configuration regular solution of the equations for the plasma is given by Eqs. (19) of $\mathrm{KT}$ in terms of an arbitrary constant $p_{1}$. $\left(J_{m}\right.$ is the $m$ th order Bessel function of first kind and $J_{m}^{\prime}$ its derivative with respect to its argument; $J_{m}$ and $J_{m}$ are here always evaluated for the argument $i \gamma \xi \eta / \zeta$.)

The equations for the vacuum are (4) through (7) of $\mathrm{KT}$ with $\boldsymbol{j}=0$ and $\epsilon=0$. For the region $\gamma>\gamma_{1}$ (outside the fixed conductor) the general regular solution is given by Eqs. (23) of $\mathrm{KT}$ in terms of arbitrary constants $B_{1}$ and $E_{1}$. ( $H_{m}$ is the $m$ th order Hankel function of first kind and $H_{m}{ }^{\prime}$ its derivative with respect to its argument; $H_{m}$ and $H_{m}{ }^{\prime}$ are here always evaluated for the argument $i \psi r$.)

In the general solution for the vacuum region, $r_{0}<r<r_{1}$ (between the plasma and the fixed conductor), we have each magnetic and electric field component given as a sum of two expressions, one the same as in Eqs. (23) of KT except for having $B_{1}$ and $E_{1}$ replaced by new constants $B_{2}$ and $E_{2}$, and the other again the same except that $B_{1}$ and $E_{1}$ are replaced by new constants $B_{3}$ and $E_{3}$ and at the same time $H_{m}$ and $H_{m}{ }^{\prime}$ are replaced by $J_{m}$ and $J_{m}^{\prime}$ (both evaluated for the argument $i \psi r$ ).

We now have the solution everywhere expressed in terms of the seven so far arbitrary constants, $p_{1}, B_{1}$, $E_{1}, B_{2}, E_{2}, B_{3}$, and $E_{3}$. We obtain relations between these from the interface and boundary conditions. From Conditions (9) through (14) of KT we obtain (only) three independent relations between $p_{1}, B_{1}$, and $E_{1}$. From Conditions (1) through (5) we obtain four independent relations between all the constants except $p_{1}$. Thus we have seven linear homogeneous equations for the seven coefficient constants. The condition that these equations have a non-trivial solution (i.e., that the determinant of their coefficients vanishes) leads to the characteristic equation, which must be satisfied by the characteristic constants of any elementary solution.

We now make the approximation of infinite light velocity by taking $\kappa_{0}=0$. We are interested only in unstable solutions, i.e., solutions for which $\omega$ has a positive real part, and we assume that $\omega$ is real. It can be proved ${ }^{5}$ (at least for $\sigma^{*}$ either zero or infinite) that this is no restriction, i.e., that all unstable modes have $\omega$ real. Introducing the dimensionless constants $\alpha_{\mathbf{P}}=B_{\mathrm{P}} / B_{0}, \alpha_{\mathrm{V}}=B_{\mathrm{V}} / B_{0}$ and the functions

$$
\begin{gathered}
W=\left(\rho_{0} / 2 p_{0}\right)^{\frac{1}{2} \gamma_{0} \omega}, \quad \Sigma=\left(2 p_{0} / \rho_{0}\right)^{\frac{1}{z}} \mu_{0} \sigma^{*} \\
K_{m}(y)=\frac{J_{m}(i y)}{i y J_{m}^{\prime}(i y)}, \quad L_{m}(y)=\frac{H_{m}(i y)}{i y H_{m}^{\prime}(i y)^{\prime}}, \\
M_{m}\left(y_{0}, y_{1}\right)=\frac{H_{m}^{\prime}\left(i y_{1}\right) J_{m}{ }^{\prime}\left(i y_{0}\right)}{H_{m}^{\prime}\left(i y_{0}\right) J_{m}^{\prime}\left(i y_{1}\right)},
\end{gathered}
$$

the (approximate) characteristic equation is

$\left[\alpha \mathrm{P}^{2} y_{0}^{2}+\left(1+\alpha \mathrm{v}^{2}-\alpha_{\mathrm{P}}^{2}\right) W^{2}\right] K_{m}(x)=1+\left(\alpha y_{0} y^{ \pm m}\right)^{2}$

$$
\times\left\{L_{m}\left(y_{0}\right)-\frac{M_{m}\left(y_{0}, y_{1}\right)\left[K_{m}\left(y_{0}\right)-L_{m}\left(y_{0}\right)\right]}{1-M_{m}\left(y_{0}, y_{1}\right)+\left(\gamma_{0} / \nu_{1}\right)} \times,\right.
$$


where the plus or minus sign is to be chosen according to whether $k$ is positive or negative, and where

$$
\begin{gathered}
y_{0}=|k| \gamma_{0}, \quad y_{1}=|k| r_{1}, \\
x=\left\{\left[y_{0}^{2}+(2 / \gamma) W^{2}\right]\right. \\
\left.\times \frac{\alpha_{\mathrm{P}}^{2} y_{0}^{2}+\left(1+\alpha \mathrm{v}^{2}-\alpha \mathrm{P}^{2}\right) W^{2}}{\alpha_{\mathrm{P}}^{2} y_{0}{ }^{2}+\left[1+\alpha \mathrm{v}^{2}+(2-\gamma) \alpha_{\mathrm{P}}^{2} / \gamma\right] W^{2}}\right\}^{\frac{1}{2}} .
\end{gathered}
$$

Numerically, $J_{m}(i y)$ and $J_{m}{ }^{\prime}(i y)$ are monotonically increasing functions of $y$, and $H_{m}(i y)$ and $H_{m}{ }^{\prime}(i y)$ are monotonically deccreasing functions of $y$. Since $y_{0}<y_{1}$ it follows that $0<M_{m}\left(y_{0}, y_{1}\right)<1$. Also, $K_{m}(y)>0$ and $L_{m}(y)<0$. We thus see that the second term in the braces on the right-hand side of Eq. (9), which term we shall denote by $U$, is negative, as is the first term $L_{m}\left(y_{0}\right)$. The left-hand side of Eq. (9) is a monotonically increasing function of $W$, at least for $W$ very small, very large, and in the neighborhood of its largest value satisfying Eq. (9), and very likely for all values. In any case, it can be proved easily that the largest value of $W$ for which the left-hand side of Eq. (9) has a prescribed value is a monotonically non-decreasing function of that prescribed value. Now characteristic Eq. (9) differs from the corresponding equation for the same equilibrium situation without the conducting sheet at $r=r_{1}$ (namely Eq. (30) of KT) only in the presence of the term $U$. It follows, consequently, that the presence of the conducting sheet has, quite generally, the effect of diminishing the rate of instability. As was to be expected, $U \rightarrow 0$ as $\Sigma \rightarrow 0$ or as $\gamma_{1} \rightarrow \infty$. In the latter case, $U \rightarrow 0$ very quickly since both functions $H_{m}{ }^{\prime}\left(i y_{1}\right)$ and $1 / J_{m}^{\prime}\left(i y_{1}\right)$ go to zero exponentially.

As pointed out earlier, Eqs. (1) through (5) are valid if the thickness $\delta$ of the shell is much less than the penetration distance, $\left(\tau / \mu_{0} \sigma\right)^{\frac{1}{2}}$, of the shell material, $\tau$ being a characteristic time for the phenomena under consideration. With the shell in the form of a cylinder of inner radius $\gamma_{1}$, we can, under rough assumptions, determine corresponding equations for the opposite limiting case when $\delta \gg\left(\tau / \mu_{0} \sigma\right)^{\frac{1}{2}}$. We take for granted that $\left(\tau / \mu_{0} \sigma\right)^{t} \ll \gamma_{1}$. We do not know a priori the distribution of induced eddy currents in the shell, but we assume, for the sake of having something definite to compute, that it is purely in the $\theta$-direction. This current distribution turns out to have a characteristic decay time of about $\frac{1}{4} \tau \gamma_{1}\left(\tau / \mu_{0} \sigma\right)^{-} \gg \tau$. Therefore there is no appreciable decay during lengths of time of interest, and the shell may be treated as a perfectly conducting sheet, with a radius perhaps exceeding $\gamma_{1}$ by something of the order of the penetration distance. Thus, with appropriate values of $r_{1}$ and $\sigma^{*}$, Eqs. (1) through (5) may still be considered to hold. The validity of this argument is of course questionable because of the arbitrariness in the choice of the current distribution, but in any case the stabilizing action of the shell must be less than it would be for a perfect conductor, which can be treated as a sheet at radius $\gamma_{1}$.

\section{APPLICATION}

We now wish to apply our theory to the stellarator. One idealization we make is to ignore the curvature of the stellarator and to treat it instead as if it were straightened out to form a right circular cylinder. Since the stellarator has finite length and the present theory deals with an infinite cylinder, one must impose some periodicity condition, to be discussed later.

Another idealization is to treat the plasma in the stellarator as a uniform plasma with all current on the surface. The effect of modifying this assumption is also discussed later.

The equilibrium situation of the theory would seem to represent reasonably well the expected conditions in the stellarator during ohmic heating if we take $B_{\mathrm{P}}=B_{\mathrm{V}}$ to represent the confining field, $2 \pi r_{0} j_{0}$ to represent the induced plasma current, and the sheet at $r=r_{1}$ to represent any coaxial cylindrical conductor, such as accelerating or confining field windings, or the stainless steel discharge tube. In the stellarator the longitudinal confining field is much larger than the maximum field produced by the plasma current, hence we have $|\alpha| \gg 1$, where $\alpha=\alpha \mathrm{P}=\alpha \mathrm{V}$. It is shown in KT that in this limiting case the only instability is for $m=1$, to which case we now confine our investigation.

It is not hard to show that if we are to obtain a real positive solution $W$ of Eq. (9) we must have $y_{0} \ll 1$ (i.e., $k$ small), $k \alpha<0, W$ not too large, and $x \ll 1$. Since $\kappa_{1}(0)=1, L_{1}(0)=-1$, and (for $y_{0}$ and $y_{1}$ small) $M_{1}\left(y_{0}, y_{1}\right) \approx r_{0}^{2} / r_{1}^{2}$, Eq. (9) becomes asymptotically $Y^{2}+W^{2}$

$$
=1-(Y-1)^{2}\left\{1+2 \Sigma W /\left[\Sigma W\left(a^{2}-1\right)+2 a\right]\right\},
$$

where

$$
Y=|\alpha| y_{0}, \quad a=\gamma_{1} / \gamma_{0} .
$$

If $\Sigma$ is finite, Eq. (11) has a positive real solution $W$ only for $0<Y<1$; for $Y \rightarrow 0$ we find $W \approx 2 a Y / \Sigma$, while for $Y \rightarrow 1$ we find $W \approx[2(1-\dot{Y})]^{\hat{1}}$. If $\Sigma=\infty$, it has a positive real solution only for $a^{-2}<Y<1$; for $Y \rightarrow a^{-2}$ we find $W \approx\left[2\left(Y-a^{-2}\right)\right]^{\frac{1}{2}}$, while for $Y \rightarrow 1$ we have $W \approx[2(1-Y)]^{\frac{1}{2}}$ as before. In any case, the maximum value of $W$ and the value of $Y$ for which it is attained satisfy, Eqs. (11) and (13) :

$$
2 Y=-2(Y-1)\left\{1+2 \Sigma W /\left[\Sigma W\left(a^{2}-1\right)+2 a\right]\right\},
$$

which is obtained from Eq. (11) by partial differentiation with respect to $Y$. From Eqs. (11) and (13) we find that

$$
Y=1-W^{2}
$$

and that $W$ is determined by

$$
W^{2}\left\{1+\Sigma W /\left[\Sigma W\left(a^{2}-1\right)+2 a\right]\right\}=\frac{1}{2}
$$

\section{UNIMPORTANCE OF EXTERNAL CONDUCTOR}

In the absence of the conducting sheet (i.e., for $a=\infty$ or $\Sigma=0$ ) Eq. (15) gives $W=1 / \sqrt{2}$ or

$$
\omega=\left(1 / r_{0}\right)\left(p_{0} / \rho_{0}\right)^{\frac{1}{2}}
$$


for the maximum rate of instability. In a stellarator we might have a tube of, say, helium plasma of about $2 \mathrm{~cm}$ radius. If the ions and electrons were both at temperature $T$ in degrees Kelvin, Eq. (16) would become

$$
\omega=3.12 \times 10^{3} T^{\wedge} \mathrm{sec}^{-1} .
$$

Since the time scale for operation of the stellarator is of the order of milliseconds, we see that for $T=10^{8}$ the instability would grow extremely fast. Even for $T=10^{4}$ an instability would be serious if its $W$ were larger than $10^{-3}$.

For the conducting sheet to have the effect of reducing the maximum $W$ to a very small value, we see from Eq. (15) that it is necessary both for $a$ to be nearly equal to unity and for $\Sigma$ to be large. Specifically, it is necessary to have

$$
a-1 \leqslant W^{2}, \quad \Sigma \geqslant W^{-3} .
$$

For the stellarator, this means that a conducting shell which is to slow up the instability enough to do any good must in the first place be extremely close to the plasma $\left(r_{1}-r_{0} \leqslant 2 \times 10^{-6} \mathrm{~cm}\right.$ for $\left.T=10^{4}\right)$. This immediately excludes all conductors except those virtually in contact with the plasma, such as stainless steel tubing. The sheet conductivities of such conductors are unlikely greatly to exceed several hundred mhos, which at $T=10^{4}$ corresponds roughly to $\Sigma=4$, whereas $\Sigma$ would have to be about $10^{9}$ to do any good; at higher temperatures the comparison is even less favorable.

Indeed, it would apparently be hopeless to slow up the instability sufficiently by external conductors even if they were designed for that purpose and it were not necessary to worry about inimical effects they might have on the normal operation of the stellarator. For instance, if one had thick walls of silver $\left(\sigma=6 \times 10^{7}\right.$ mho/meter) arbitrarily close to the plasma, the silver could be treated as a perfectly conducting sheet at a radius greater than the plasma radius $r_{0}$ by approximately the penetration distance of the silver, and computation shows that, even for a plasma temperature as low as $0.3^{\circ} \mathrm{K}$, the instability would then $e$-fold in a millisecond.

\section{PERIODICITY CONDITION}

Now that we have seen that external conductors have a negligible effect on the instability, we turn to an examination of the restrictions on perturbations imposed by the geometry of the stellarator. We wish to treat the stellarator tube as if it were straightened out to a right circular cylinder. Put another way, we wish to define coordinates $\gamma, \theta, z$ in the tube which locally are approximately cylindrical coordinates and in terms of which the (inner) surface of the tube is approximately the surface $r=r_{0}$. It is natural to choose the curve $r=0$ to be the magnetic axis of the stellarator (i.e., the magnetic line of force which closes upon itself after one traversal of the length of the tube); $r, \theta$ to be polar coordinates in each cross section of the tube; $z$ to be constant on each cross section; and $z$ to be the arc length along the curve $r=0$ (with the sign of $d z$ chosen so as to make $r, \theta, z$ a right-handed coordinate system). It remains only to determine the relative rotation of the polar coordinates in different cross sections, i.e., to determine the direction $\theta=0$ (say) in each cross section. Choosing an arbitrary vector at $r=0$ lying in one cross section to give the direction $\theta=0$ there, we consider a parallel vector at the point $r=0$ of a neighboring cross section. This parallel vector does not in general lie in the neighboring cross section, but we may choose its projection thereon as the direction $\theta=0$. In this way the direction $\theta=0$ may be carried successively around the length of the stellarator. That this is the natural method of relating the values of $\theta$ in different cross sections may be seen in several ways. One way is to observe that what we have done is equivalent to requiring that the coordinates $\gamma$ and $\theta$ be invariant when the $z$ cross section is projected onto the $z+d z$ cross section in the direction of the magnetic axis; the resultant values of $r$ and $\theta$ in the $z+d z$ cross section do not exactly constitute polar coordinates, but the deviation is of the order $d z^{2}$ and is therefore negligible. Another way is to observe that the lines of force of the confining magnetic field approximate to curves of constant $r$ and $\theta$.

The "cylindrical" coordinates we have defined in the tube are not single-valued functions of position (except for $r$, which is the distance from the magnetic axis). If we follow the values of $\theta$ and $z$ along a closed curve which goes once around the length of the stellarator in the direction of positive $d z$, we find that when we have returned to the starting point, $z$ has increased by the length $L$ of the magnetic axis and $\theta$ has increased by a definite angle $\iota$ depending only on the geometry of the stellarator (and not at all on the starting point or the particular curve chosen). This is called the rotational transform angle.

It can be shown by standard methods of the differential geometry of space curves that $-\imath$ is equal to the integral of the torsion of the magnetic axis with respect to its arc length, once around the stellarator. (The torsion of a curve is the negative of the rate of rotation, with respect to arc length, of the osculating plane, i.e., of the plane determined by the tangent and the radius of curvature. The positive direction of rotation is determined by the right-hand rule from the direction along the curve in which the arc length is taken as increasing.) For stellarators of twisted figure-8 shape, ${ }^{4}$ let $\phi$ be the angle through which each end of a plane Figure 8 must be rotated to arrive at that shape, the positive direction of rotation for each end being clockwise as seen from beyond that end. It is then easily seen that

$$
\iota=-4 \phi \text {. }
$$

Now $(r, \theta, z)$ and $(r, \theta+\iota, z+L)$ represent the same point in the tube, so in our perturbation theory we can allow only elementary solutions for which $m \imath+k L$ is an integral multiple of $2 \pi$. We recall that the unstable 
perturbations we are concerned with have $m=1$, $k \alpha<0,0<Y<1$. Since $Y=|\alpha k| r_{0}$, we see that there is one such allowable perturbation for each (positive or negative) integer $h$ satisfying

$$
0<\left(\alpha \gamma_{0} / L\right)(\iota+2 \pi h)<1 .
$$

Thus the condition that no instability be allowable is that

$$
\alpha(\iota+2 \pi h) \geqslant L / r_{0},
$$

where $h$ is that integer which gives the left-hand side of Eq. (21) its smallest positive value.

It is clear that the stability criterion (21) depends not only on the magnitude of $\alpha$ but also upon its sign, unless $\iota$ happens to be an integral multiple of $\pi$. We note that $\alpha$ is positive or negative accordingly as the induced longitudinal plasma heating current has the same or the opposite direction as the longitudinal confining magnetic field.

Condition (21) is more conveniently expressed, for application, in terms of the plasma current $I=$ $2 \pi r_{0} j_{0} *=2 \pi r_{0} B_{0} / \mu_{0}$. Since $\alpha=B_{\nabla} / B_{0}$, Eq. (21) may be written

$$
\left(B_{\mathrm{V}} / I\right)(\iota+2 \pi h) \geqslant \mu_{0} L / 2 \pi r_{0}^{2} .
$$

\section{CURRENT DISTRIBUTION EFFECTS}

Some longitudinal current distributions more general than the purely surface current case first considered above are treated elsewhere ${ }^{6}$ by means of the energy principle. ${ }^{5} \mathrm{We}$ quote the results without the negligible complications of an external conductor. The fluid pressure $p$ is taken to be zero, $B_{z}$ is again taken to be much larger than $B_{\theta}$, and the condition for cylindrically symmetric equilibrium,

$$
\frac{1}{2} \frac{\partial}{\partial r}\left(B_{\theta}^{2}+B_{z}^{2}\right)+B_{\theta}^{2} / r=0
$$

is satisfied for $B_{\theta}$ any function of $r$ by choosing $B_{z}$ to be the appropriate nearly constant function. The conclusion then is that there is one allowable mode of instability for each (positive or negative) integer $h$ satisfying

$$
1 / Z<\left(\alpha c r_{0} / L\right)(m \iota+2 \pi h)<m
$$

where $Z>0$ depends on the function $B_{\theta}$, i.e., on the distribution of current $j_{z}$, and on $m$. This is the generalization of Eq. (20).

For $m=0$, there is clearly no instability. For $m=1$, $Z$ becomes infinite, independently of the current distribution, and thus Eq. (24) reduces to Eq. (20).

For $m>1, Z$ is given as a function of a positive exponent $\nu$ in Fig. 2 for $j_{z}$ proportional to $1-\left(r / r_{0}\right)^{\nu}$ and also to $\left(r / r_{0}\right)^{\nu}$. The first type of distribution with $\nu=\infty$ and the second with $\nu=0$ are identical, both representing uniform current, and have $Z=1 /(m-1)$.

For both types of distribution $Z$ is greater than $1 / m$ and increases monotonically with $\nu$. We see that for each $m>1$ there are thus always ranges of values for $\alpha$ for which there is instability. These ranges increase with $\nu$.
As $\nu \rightarrow \infty$ the second type of distribution approaches the sheet current case considered earlier (and $Z$ approaches $(m+1) /(m-1) m)$. The non-vanishing of the ranges of instability in the limit seems somewhat paradoxical because the limiting sheet current case is stable for $m>1$. The resolution is probably that the unstable perturbations become stabilized by nonlinear effects at smaller and smaller amplitudes as one goes to the limit.

These results have applied when the plasma is surrounded by vacuum. If, instead, it is surrounded by pressureless plasma, but the equilibrium fields are the same, then the results for $m=0$ and $m=1$ are the same, but for $m \geqslant 2$ there is now complete stability. It seems uncertain whether vacuum or pressureless plasma is the better approximation to conditions in the region between the main plasma and the tube wall in a stellarator.

\section{EXPERIMENTAL RESULTS}

It has been shown in the previous sections that a figure-8 shaped stellarator should exhibit an $m=1$ instability for currents greater than a critical value determined by Eq. (22). The magnitude of this critical current depends on the geometry of the system (through $\iota$, the rotational transform angle, and $L$, the axial length), the plasma cross-sectional area (through $\gamma_{0}$, the radius), $B_{\mathrm{V}}$ the magnitude of the longitudinal confining magnetic field, and on whether the current direction is along or opposite to the magnetic field. It is interesting to note that the critical current in either of the two directions is just sufficient to cause a resultant rotation of either zero or $2 \pi$ in the magnetic lines of force just outside the plasma (i.e., to make the lines of force close on themselves once around the machine). This result is independent of the form of current distribution.

The quantities $\iota, I, B_{\mathrm{V}}$ and $L$ are all easily measurable. If all the lines of force were exactly parallel to the walls of the discharge tube, then $r_{0}$ would simply be the discharge tube radius. If this is not the case, $r_{0}$

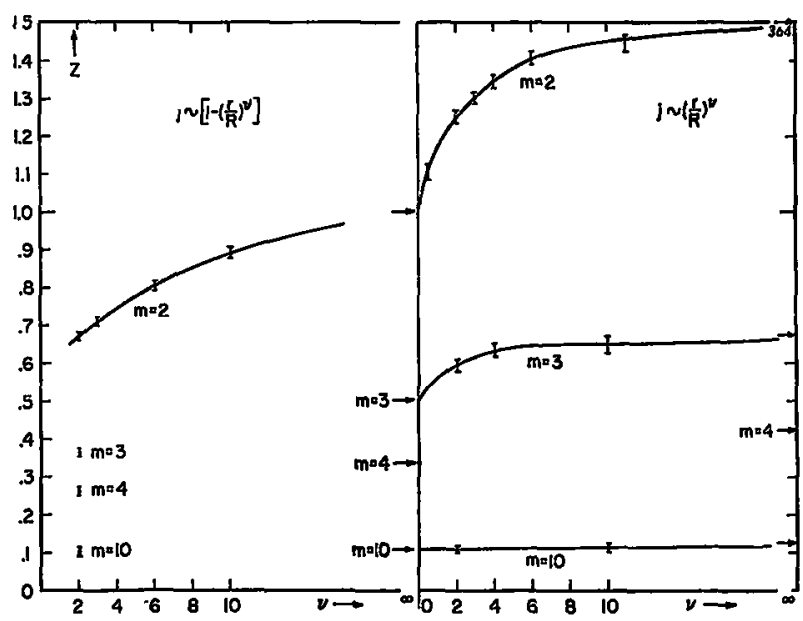

Figure 2. Limit of range of instability for axlal current distributions $j \sim 1-(r / R)^{2}$ and $j \sim(r / R)^{2}$ 

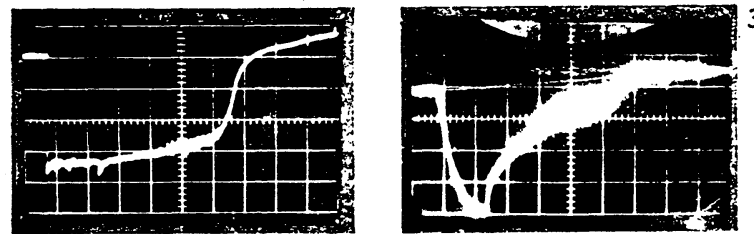

364

$0.050 \mathrm{VOLTS} / \mathrm{CM}$

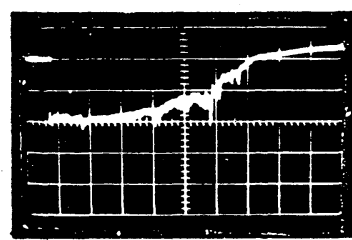

0.057

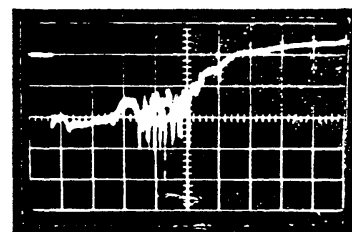

0.062

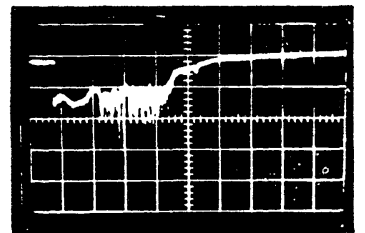

0.072

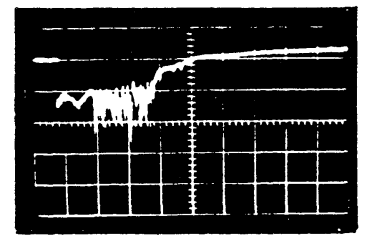

0.078

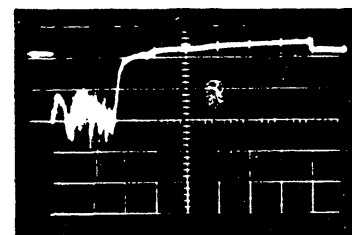

0.11

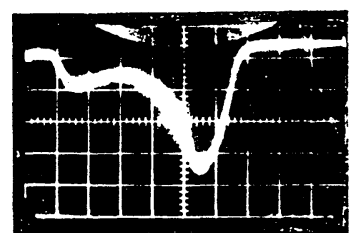

500

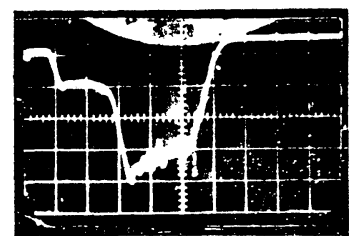

500

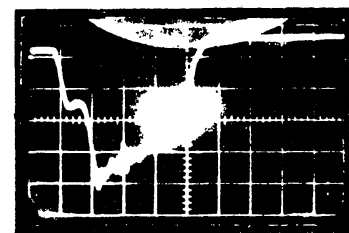

500

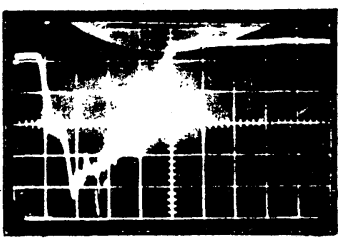

500

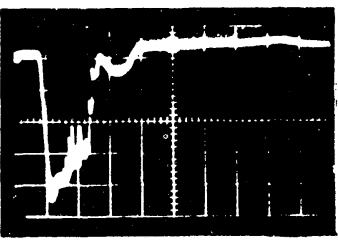

500

C

where $\eta=0.911 \pi$ and $1.089 \pi$ for the two possible directions of current and $B_{\mathrm{V}}, r, L$ and $\mu_{0}$ are all in MKS units.

A The observational results to be expected as a consequence of this instability are not entirely obvious. The observations on the B-1 Stellarator where the effects seem particularly apparent will first be described in some detail, followed by a summary of similar evidence from other devices.

Table 1. Some Stellarator Characteristics

\begin{tabular}{llllll}
\hline $\begin{array}{c}\text { Stellarator } \\
\text { model }\end{array}$ & $L$ & $\begin{array}{c}\text { Tube } \\
\text { inside } \\
\text { radius }\end{array}$ & $\begin{array}{c}\text { Aperture } \\
\text { radius }\end{array}$ & $\begin{array}{c}\text { Critical } \\
\text { current at } \\
10 \text { kilogauss }\end{array}$ \\
\hline B-1 & $196^{\circ}$ & $450 \mathrm{~cm}$ & $2.2 \mathrm{~cm}$ & $1.6 \mathrm{~cm}$ & 810 or 970 amps \\
B-2 & $196^{\circ}$ & 600 & 2.2 & 1.6 & 610 or 730 \\
B-3 & $196^{\circ}$ & 600 & 2.2 & 2.0 & 950 or 1140 \\
\hline
\end{tabular}

The ohmic heating electric field is usually applied in the form roughly of a square wave of adjustable amplitude by means of a transformer which links the discharge tube. A more complete description of the characteristics of the stellarator is given by Coor, et al. ${ }^{7}$ The duration time is self-limited by saturation of the transformer. The plasma current and applied electric fields are displayed as functions of time on oscilloscopes. Figure 3 shows these data for six different $E$-fields, applied to a helium discharge at an initial pressure of $6 \times 10^{-4} \mathrm{~mm} \mathrm{Hg}$. The oscilloscope sweep speed is 1 millisecond per division from left to right and the initially applied electric field in $\mathrm{v}$ per $\mathrm{cm}$ is given in each case. The field gradually falls (because of

E the partial discharge of a capacitor bank) and fluctuations appear because of plasma inductance effects. As successively higher electric fields are applied, the current rises more rapidly and (in the first four cases) to a higher peak value. However, for fields above about $0.06 \mathrm{v} / \mathrm{cm}$ there is very little dependency of peak current on applied voltage as shown in Fig. 4, which shows peak current plotted against applied electric field at various gas pressures. The current essentially reaches a plateau, the level of which is roughly independent of the pressure. As shown in the figure, the plateau value of current agrees with that predicted from Eq. (25). Similar sets of data taken at other values of confining field produce similar effects at current levels proportional to the magnetic field in quantitative agreement with prediction. These are the solid circles in Fig. 5. However, the most striking effect is that of reversing the direction of the current with respect to the magnetic field. These data are plotted as open circles in Fig. 5. The ratio of slopes of the two lines is 1.22 . On the basis of the twist angle of B-1, one would expect a ratio of 1.19 . This difference is well within experimental error.

Another point of interest is that whenever the current rises above this critical limiting value, the current and voltage become quite noisy as may be seen in Fig. 3, and large amounts of impurities appear in the discharge.?

Further verification is offered by the fact that the

$$
I=B_{\mathrm{V}} 2 \pi r_{0}^{2} \eta / \mu_{0} L,
$$

pertinent quantities is given in Table 1 .

which becomes, on solving for $I$ 


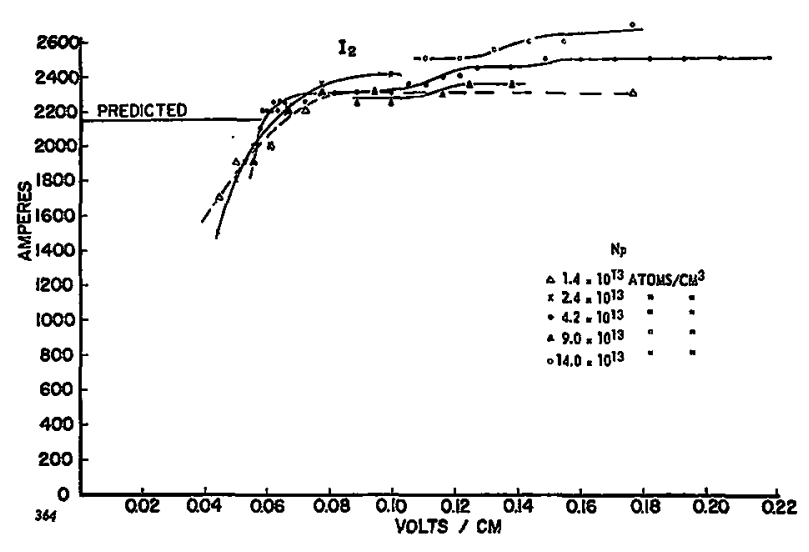

Figure 4. Peak plasma current as a function of applied electric fleld at various pressures in B-1

critical value of current is the same in a hydrogen discharge as in a helium discharge.

In all cases it is possible to drive the current well above the critical current if a high enough electric field is applied. Figure 6 shows, for example, a plot of peak current $v s$. ohmic heating field for 3 different values of magnetic confining field in the B-2 Stellarator. There is once more a definite leveling off, at a current consistent with prediction, but for high ohmic heating fields the current does continue to rise. However, in this case a slight step or irregularity appears at approximately the critical current (shown by the dotted lines in Fig. 6).

In the case of B-3, much more care was taken in alignment of the field coils and the discharge tube. As a result, the aperture area as measured by the electron-gun is $13 \mathrm{~cm}^{2}$ as compared with about $8 \mathrm{~cm}^{2}$ in both B-1 and B-2. Correspondingly higher plateau currents are expected and are observed in this case up to a magnetic confining field of 53,000 gauss. The dis-

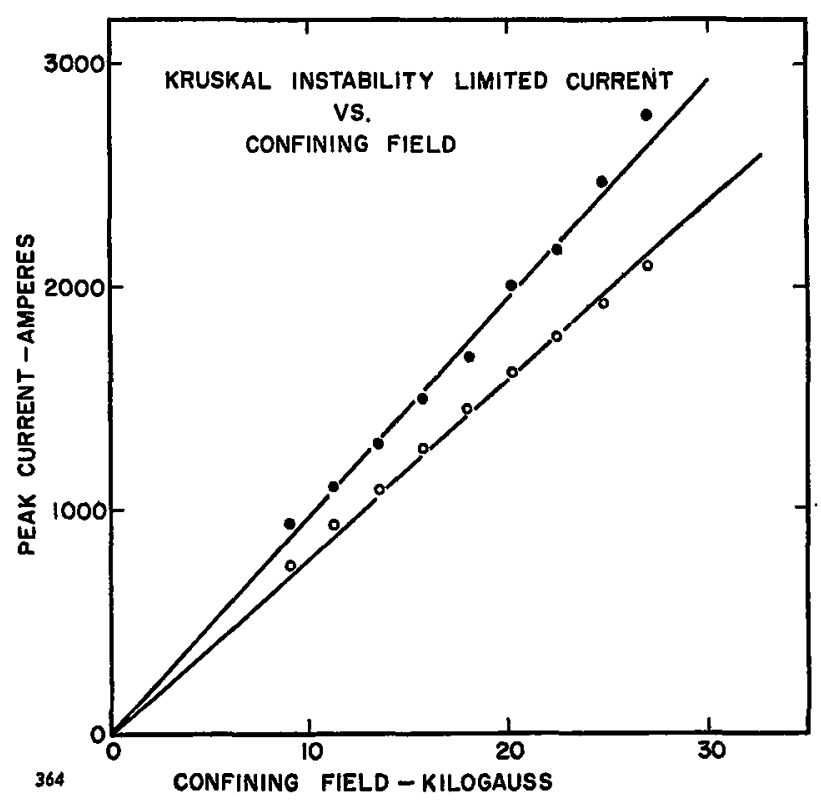

Figure 5. Peak plasma current as a function of confining field in B-1. The two curves are for plasma current in opposite directions

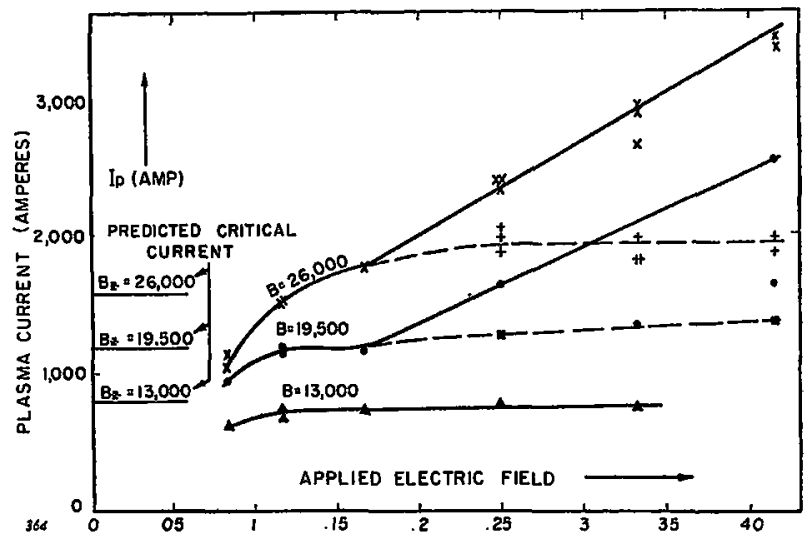

Figure 6. Peak plasma current as a function of applied electric field in B-2

charge tubes in all the devices previously mentioned are stainless steel with bakable vacuum systems which may be pumped down to pressures of the order of $10^{-10} \mathrm{~mm} \mathrm{Hg}$.

B-1 was formerly operated with relatively "dirty" walls of stainless steel, and later of Pyrex, for which base pressures of only about $10^{-5} \mathrm{~mm} \mathrm{Hg}$ were possible. The metal tube required about 4 times as much electric field to get initial breakdown and showed very little evidence of the current leveling off. In both these respects, on the other hand, the "dirty" glass system was quite similar to the "clean" metal system.

Clearly there is very satisfactory agreement between theory and experiment with regard to the $m=1$ mode of instability. However, there is no experimental indication of the existence of the higher $m$ modes. One possible explanation of this is that the region between the main body of plasma and the tube wall might contain enough charged particles to act as a good conductor, i.e., as a pressureless plasma, in which case the higher $m$ modes would be stable. It is of course quite possible that higher $m$ modes of instability are present but produce effects less easily observable than those due to the $m=1$ mode. For instance, they might merely distort the original equilibrium configuration into a new, not greatly shifted, stable configuration. This would be consistent with the proposed resolution of the paradox in the previous section. It seems highly plausible that for long wavelength modes with $m \neq 1$, non-linear terms become significant when the displacement becomes comparable with the plasma radius, whereas for long wavelength $m=1$ modes, the linearized perturbation theory remains valid until the displacement becomes comparable with the wave-length.

\section{REFERENCES}

1. M. D. Kruskal and J. L. Tuck, The Instability of a Pinched Fluid with a Longitudinal Magnetic Field, Proc. Roy. Soc. $A$, in press (1958).

2. M. D. Kruskal and M. Schwarzschild, Some Instabilities of a Completely Ionized Plasma, Proc. Roy. Soc., A223, p. 348 (1954). 
3. L. Spitzer, The Stellarator Concept, P/2170, Vol. 32, these Proceedings.

4. M. D. Kruskal, Large-Scale Plasma Instability in the Stellavator, AEC Report No. NYO-6045 (PM-S-12, 1954).

5. I. B. Bernstein, E. A. Frieman, M. D. Kruskal and R. M. Kulsrud, An Energy Principle for Hydromagnetic Stability Problems, Proc. Roy. Soc., A244, 17 (1958).
6. J. L. Johnson, C. R. Oberman, R. M. Kulsrud and E. A. Frieman, On the Stability of Hydromagnetic Equilibria with Varying Rotational Transform, AEC Report No. NYO-7904 (PM-S-34, 1958).

7. T. Coor, S. P. Cunningham, R. A. Ellis, M. A. Heald and A. Z. Kranz, Experiments on the Ohmic Heating and Confinement of Plasma in a Stellavator, $\mathrm{P} / 362$, Vol. 32, these Proceedings. 